\title{
Crossover from percolation to diffusion
}

\author{
D. N. Tsigankov, A. L. Efros \\ Department of Physics, University of Utah, Salt Lake City UT 84112 USA
}

\begin{abstract}
A problem of the crossover from percolation to diffusion transport is considered. A general scaling theory is proposed. It introduces phenomenologically four critical exponents which are connected by two equations. One exponent is completely new. It describes the increase of the diffusion below percolation threshold. As an example, an exact solution of one dimensional lattice problem is given. In this case the new exponent $q=2$.
\end{abstract}

Percolation theory is often used to describe transport properties of disordered systems with a large disorder. The typical problems are random mixture of conducting and nonconducting elements, or hopping conduction [1]. The regular percolation theory assumes that the random elements do not change their positions with time so that the percolation paths do not change their spatial locations. The low-temperature electron transport is one of examples where this assumption is not valid. Due to electron-electron interaction the random potential persistently and substantially changes with time [2,3], which may affect conductivity near the metal-insulator transition. Such problems appear outside solid state physics as well. The class of this problem is known as dynamical percolation. They have been studied theoretically using effective medium approximation (See e.g. Ref. [4] and references therein). One dimensional problems of this type have been considered for some models without any approximations [5].

We concentrate here on the case when the diffusion through conducting media is drastically faster then the fluctuations of the positions of the conducting and non-conducting elements. In this case the transition from diffusion to percolation mechanism has all features 
of the phase transition and it can be characterized by critical exponents. In this paper we introduce a set of these exponents and establish relation between them. For illustration we present an exact solution of one dimensional lattice problem.

Thus, we consider here a random mixture of the conducting and non-conducting elements which can change there positions but very slowly. In this situation the resulting diffusion (or conductivity) of the particles is non-zero at any small fraction $p$ of the conducting elements. The mechanism of this diffusion is as follows. A particle can diffuse only in the conducting medium. To move from one conducting element to the other it is waiting until another conducting element comes to the element where it resides. At this moment of time a particle is able to do a next move. This is the simple diffusion which is characterized by some slow waiting time $\tau_{s}$. The diffusion inside conducting medium is characterized by much faster time $\tau_{f}$. When the fraction of conducting elements $p$ becomes close to the percolation threshold $p_{c}$, but it is less than $p_{c}$ the resulting diffusion increases as $1 /\left(p_{c}-p\right)^{q}$, where $q$ is a novel critical exponent. This happens because the conducting clusters become large. But since they are disconnected the particle should wait to jump from one of them to the other. Finally, when $p-p_{c}$ is positive and it becomes larger than the width of some critical region, the diffusion is described by a regular percolation law $D \sim\left(p-p_{c}\right)^{t}$ and slow motion of the conducting elements is not important. To describe this physics one can use the same scaling arguments as for the problem of frozen mixture of elements with large and small conductivity [6,7]. Note that the new problem is not equivalent to the old one, so that the exponent $q$ might be different. The scaling hypothesis is valid in the proximity of the percolation threshold so that $|X| / p_{c} \ll 1$, where $X=p-p_{c}$. In this region the scaling hypothesis can be written in the form

$$
d=h^{s} \psi\left(\frac{X}{h^{m}}\right)
$$

Here $d=D / D_{f}, h=D_{s} / D_{f}$, where $D_{f}=a^{2} / 2 \tau_{f}$ is the diffusion coefficient in the conducting medium, $a$ is a characteristic length which depends on the model, $D_{w}=a^{2} / 2 \tau_{s}$, and $\psi(Z)$ is some analytical function at all real values of its argument, $-\infty<Z<\infty$. We assume that 
$h \ll 1$ and that $\psi(0)=1$.

Eq. (1) contains two independent critical exponents. The meaning of the exponent $s$ is that $d=h^{s}$ at $p=p_{c}$. The exponent $m$ describes the width of the critical region $\left|p-p_{c}\right|=h^{m}$ between the percolation and diffusion.

All the critical exponents which describe $d(X)$ can be related to $s$ and $m$. At $X>0$ and $X \gg h^{m}$ the slow changes of the percolation paths are not important. Thus, we have the diffusion on the percolation cluster. If the system is larger than the correlation radius of the percolation cluster, the diffusion coefficient is related to the conductivity by the Einstein relation. Then one has $d \sim X^{t}$, where the exponent $t$ is a usual percolation exponent which describes the conductivity above the percolation threshold. To get this result from Eq. (1) one should assume that $m=s / t$. At $X<0$ and $|X| \gg h^{m}$ we expect that $d \sim h /(|X|)^{q}$. This gives $s+m q=1$. Finally we get two connections between four exponents $s, q, t, m$, namely $q=t\left(s^{-1}-1\right)$ and $m=s / t$.

As an example, we consider below an exactly soluble one dimensional site or bond model on a lattice. At the beginning we consider the site model. The sites may be white and black with the fractions $p$ and $1-p$ respectively. The particle may occupy the white sites only and it is able to jump at the nearest site, if this site is white, during the time $\tau_{f}$. The configuration of the white and black sites slowly changes with time. This change can be introduced by many ways. We consider the simplest one. After each time interval $\tau_{s}$, which is called the renewal time, the configuration of all sites completely changes preserving the same $p$, while the particle remains at the same white site. We assume that $\tau_{s} \gg \tau_{f}$. Since $p_{c}=1$ in the one-dimensional case, our solution may only illustrate the increase of the diffusion coefficient $D \sim(1-p)^{q}$ and the width of the critical region $|X|$, where it deviates from this law and tends to the value $D_{f}=a^{2} / 2 \tau_{f}$ which is reached at $p=1$. Since the diffusion of a particle within the time interval $\tau_{s}$ is completely independent of the diffusion during other time intervals, one has

$$
\overline{r^{2}(t)}=\overline{\left(r_{1}\left(t_{1}\right)+r_{2}\left(t_{2}\right)+\ldots+r_{N}\left(t_{N}\right)\right)^{2}}=\overline{r_{1}^{2}\left(t_{1}\right)}+\overline{r_{2}^{2}\left(t_{2}\right)}+\ldots+\overline{r_{N}^{2}\left(t_{N}\right)}
$$


It follows that

$$
D=\lim _{t \rightarrow \infty} \frac{r^{2}(t)}{2 t}=\frac{\overline{r^{2}\left(\tau_{s}\right)}}{2 \tau_{s}}
$$

where $\overline{r^{2}\left(\tau_{s}\right)}$ is averaged over all possible initial position of a particle.

Now our strategy is as follows. We assume first that the clusters of the white sites are not very large so that the particle, which change position during the time $\tau_{f}$ crosses them to and fro many times during the time $\tau_{s}$. In this approximation we find diffusion coefficient $D_{1}$. For the larger clusters we use the continuum approximation, which is valid at $1-p \ll 1$ only, and find $D_{2}$. This coefficient gives the correct value at $p=1$. These two approximations have a large region of overlap if

$$
\frac{\tau_{f}}{\tau_{s}} \ll 1
$$

Matching $D_{1}$ and $D_{2}$ in the overlap region we find the result which is exact if Eq. (四 is fulfilled.

To calculate $D_{1}$ we assume that the particle crosses each cluster many times during the time $\tau_{s}$. Then the average quadratic displacement $R^{2}(S)$ is independent of $\tau_{s}$ and it is given by the equation

$$
R^{2}(S)=\frac{a^{2}}{S^{2}} \sum_{n=1}^{S} \sum_{k=1}^{S}(n-k)^{2}=\frac{a^{2}}{6}\left(S^{2}-1\right) .
$$

The probability that a particle is within a cluster of $S$ white sites is

$$
w_{s}=(1-p)^{2} p^{S-1} S
$$

By averaging $R^{2}(S)$ over all the clusters one gets

$$
\overline{r^{2}\left(\tau_{s}\right)}=\frac{a^{2}}{6}\left((1-p)^{2} \sum_{S=1}^{\infty} p^{S-1} S\left(S^{2}-1\right)=\frac{a^{2} p}{(1-p)^{2}}\right.
$$

Thus,

$$
D_{1}=\frac{\overline{r^{2}\left(\tau_{s}\right)}}{2 \tau_{s}}=\frac{D_{s} p}{(1-p)^{2}}
$$


where $D_{s}=a^{2} / 2 \tau_{s}$. Note that Eq. (8) has been obtained by Druger et al. [5]. The Eq.(8) is valid if $\overline{r^{2}\left(\tau_{s}\right)}<<D_{f} \tau_{s}$. It is fulfilled if

$$
\frac{p}{(1-p)^{2}} \ll \frac{\tau_{s}}{\tau_{f}}
$$

At $p \ll 1$ one gets

$$
D_{1}=\frac{a^{2} p}{\tau_{s}}
$$

To calculate $D_{2}$ we use the continuous approximation which is valid when the size of white clusters $S \gg 1$. This is true if $1-p \ll 1$. In this approximation one should solve the diffusion equation

$$
\frac{\partial u(x, t)}{\partial t}=D_{f} \frac{\partial^{2} u(x, t)}{\partial x^{2}}
$$

assuming zero current at the beginning and at the end of the cluster $(x=0, L)$

$$
\left.\frac{\partial u(x, t)}{\partial x}\right|_{x=0, L}=0
$$

The initial condition has a form

$$
\left.u(x, t)\right|_{t=0}=\delta\left(x-x_{0}\right)
$$

where $x_{0}$ is a random point within the interval $(0, L)$.

The solution has a form

$$
u(x, t)=\frac{1}{L}+\sum_{n=1}^{\infty} \cos \left(\frac{\pi n x_{0}}{L}\right) \cos \left(\frac{\pi n x}{L}\right) \exp \left(-\frac{\pi^{2} n^{2}}{L^{2}} D_{f} t\right)
$$

The mean squared displacement with respect to the initial position $x_{0}$ is

$$
\begin{gathered}
\overline{r_{L}^{2}\left(x_{0}, t\right)}=\int_{0}^{L}\left(x-x_{0}\right)^{2} u(x, t) d x= \\
=\frac{L^{2}}{3}-\left(L-x_{0}\right) x_{0}+4 L^{2} \sum_{n=1}^{\infty} \frac{\cos \left(\frac{\pi n x_{0}}{L}\right)}{(\pi n)^{2}}\left[\frac{x_{0}}{L}+(-1)^{n}\left(1-\frac{x_{0}}{L}\right)\right] \exp \left(-\frac{\pi^{2} n^{2}}{L^{2}} D_{f} t\right)
\end{gathered}
$$

Finally, taking the average over the initial positions $x_{0}$ on the cluster, we get the time dependence of the mean square displacement of the particle on the cluster of the size $L$. 


$$
\begin{gathered}
\overline{r_{L}^{2}(t)}=\frac{1}{L} \int_{0}^{L} \overline{r^{2}\left(x_{0}, t\right)} d x_{0}= \\
=\frac{L^{2}}{6}-L^{2} \sum_{n=0}^{\infty} \frac{1}{\left(\frac{\pi}{2}(2 n+1)\right)^{4}} \exp \left(-\frac{\pi^{2}(2 n+1)^{2}}{L^{2}} D_{f} t\right)= \\
=\frac{16}{\pi^{4}} L^{2} \sum_{n=0}^{\infty} \frac{1}{(2 n+1)^{4}}\left[1-\exp \left(-\frac{\pi^{2} n^{2}}{L^{2}} D_{f} t\right)\right]
\end{gathered}
$$

One can see from Eq. (16) that for relatively small times, $t<<L^{2} / D_{f}$, the displacement $\overline{r_{L}^{2}(t)}$ grows as $2 D_{f} t$, just as for the normal diffusion, while at large times the value $\overline{r_{L}^{2}(t)}$ tends to the asymptotic value $L^{2} / 6$ indicating that the particle crosses the cluster many times.

To find the diffusion coefficient $D_{2}$ one has to average $\overline{r_{L}^{2}\left(\tau_{s}\right)}$ over all clusters with the distribution function given by Eq. (6).

In the region $(1-p)<<1$ one can substitute summation over the cluster sizes by integration to get

$$
\begin{gathered}
\overline{r^{2}\left(\tau_{s}\right)}=\frac{(1-p)^{2}}{a^{2}} \int_{0}^{\infty} \overline{r_{L}^{2}\left(\tau_{s}\right)} L \exp \left(\frac{L}{a} \ln p\right) d L= \\
=\frac{a^{2}}{(1-p)^{2}}-\frac{16}{\pi^{4} a^{2}}(1-p)^{2} \sum_{n=0}^{\infty} \frac{1}{(2 n+1)^{4}} \int_{0}^{\infty} L^{3} \exp \left(\frac{L}{a} \ln p-\frac{\pi^{2} n^{2} a^{2}}{2 L^{2}} \frac{\tau_{s}}{\tau_{f}}\right) d L
\end{gathered}
$$

The diffusion coefficient $D_{2}$ can be represented in a form

$D_{2}=\frac{\overline{r^{2}\left(\tau_{s}\right)}}{2 \tau_{s}}=\frac{16 D_{s}}{\pi^{4} a^{4}}(1-p)^{2} \sum_{n=0}^{\infty} \frac{1}{(2 n+1)^{4}} \int_{0}^{\infty} L^{3} \exp \left(\frac{L}{a} \ln p\right)\left[1-\exp \left(-\frac{\pi^{2} n^{2} a^{2}}{2 L^{2}} \frac{\tau_{s}}{\tau_{f}}\right)\right] d L$

or

$D_{2}=\frac{16 D_{s}}{\pi^{4}(1-p)^{2}} \sum_{n=0}^{\infty} \frac{1}{(2 n+1)^{4}} \int_{0}^{\in f t y} x^{3} \exp (-x)\left[1-\exp \left(-\frac{\pi^{2}(2 n+1)^{2}}{2 x^{2}} \frac{\tau_{s}}{\tau_{f}}(\ln p)^{2}\right)\right] d x$

where $x=-\frac{L}{a} \ln p$.

Introducing the dimensionless parameter $A=\pi^{2}(\ln p)^{2} \tau_{s} / 2 \tau_{f}$ and changing the integration variable $x=b_{n} \sqrt{z}$, where $b_{n}=\sqrt{A}(2 n+1)$ one can perform the summation over $n$ to get

$$
D_{2}=\frac{a^{2} A}{\pi^{2} \tau_{f}} \int_{0}^{\infty} \frac{z(1-\exp (-1 / z)) d z}{\sinh (\sqrt{A z})}
$$


At $A \gg 1$ one can neglect the exponent in the nominator of Eq.(20). Then

$$
D_{2}=D_{s} /(1-p)^{2}
$$

At $A \ll 1$ one can expand this exponent to get $D_{2}=D_{f}$. One can also get the next term at small $A$. Thus,

$$
D_{2}=D_{f}\left[1-\frac{2 \sqrt{A}}{p i^{2}} \int_{0}^{\infty} \sqrt{z}\left(\frac{1}{z}-1+\exp \left(-\frac{1}{z}\right)\right) d z\right] .
$$

Calculating the integral in Eq. (22) one gets

$$
D_{2}=D_{f}(1-0.479 \sqrt{A})=D_{f}\left(1-1.06 \sqrt{\tau_{s} / \tau_{f}}(1-p)\right)
$$

at $A \ll 1$.

Note that the linear dependence in $1-p$ means that the correction is proportional to the

ratio of the mean displacement of a particle $\sqrt{D_{f} \tau_{s}}$ during the time $\tau_{s}$ to the typical size of a white cluster $(1-p)^{-1}$.

The scaling arguments in some different form than Eq. (11) can be applied to the onedimensional case as well. It can be written in a form

$$
D_{2}=D_{f} F\left(\frac{1-p}{h^{m}}\right)
$$

where $h=\tau_{f} / \tau_{s}$. It has the same form as Eq. (1) except that $s=0$. This relation is defined at $p \leq 1$ and $1-p \ll 1$. In the same way as before, we put $F(0)=1$. At large values of $(1-p) / h^{m}$ one has $D \sim h /(1-p)^{q}$. It follows that $m q=1$ which is analogous to the relation $s+m q=1$ in the many-dimensional case. It follows from Eq.(21) that $q=2$. At $1-p \ll 1$ one can write $A=\pi^{2}(1-p)^{2} \tau_{s} / 2 \tau_{f}$. Then it follows that $m=1 / 2$ in agreement with scaling relation. The exponent $t$ is not defined in the one-dimensional case.

Finally we present the solution for the effective diffusion coefficient $D$ which is valid at all values of $p$ within the interval $0 \leq p \leq 1$. It has been shown that $D=D_{1}$ if $p /(1-p)^{2} \ll \tau_{s} / \tau_{f}$. On the other hand $D=D_{2}$ if $(1-p) \ll 1$. Thus, the two approximation have a wide region of overlap $\left(p \tau_{f} / \tau_{s}\right)^{0.5} \ll(1-p) \ll 1$. In this region $D_{1}=D_{s} p /(1-p)^{2}$ 
and $D_{2}=D_{s} /(1-p)^{2}$. Thus one can get a result which is exact everywhere if $\tau_{s} \gg \tau_{f}$. This result is $p D_{2}$. Finally,

$$
D=\frac{a^{2} A p}{\pi^{2} \tau_{f}} \int_{0}^{\infty} \frac{z(1-\exp (-1 / z)) d z}{\sinh (\sqrt{A z})}
$$

This result has been derived above for the site problem. One can see, however, that it remains unchanged for the bond problem as well.

The diffusion coefficient $D / D_{f}$ as given by Eq.(25) is plotted in Fig. 1 versus $(1-p)$ in the double logarithmic scale at different values of $\tau_{s} / \tau_{f}$. The function $(1-p)^{-2}$ is also shown there as a dot-dashed line. One can see that at large values of $\tau_{s} / \tau_{f}$ the curves have wide regions which are parallel to this line. In these regions the diffusion coefficient increases as $(1-p)^{-2}$

Finally, we have presented a novel problem which fills a gap between diffusion and percolation in case when the motion of the random media is very slow. We have considered the de transport only. It would be interesting to study a frequency dependent transport in the same conditions.

We thank B. D. Laikhtman for a fruitful discussion. This work was supported by the US-Israel Binational Science Foundation, grant 9800097. 


\section{FIGURES}

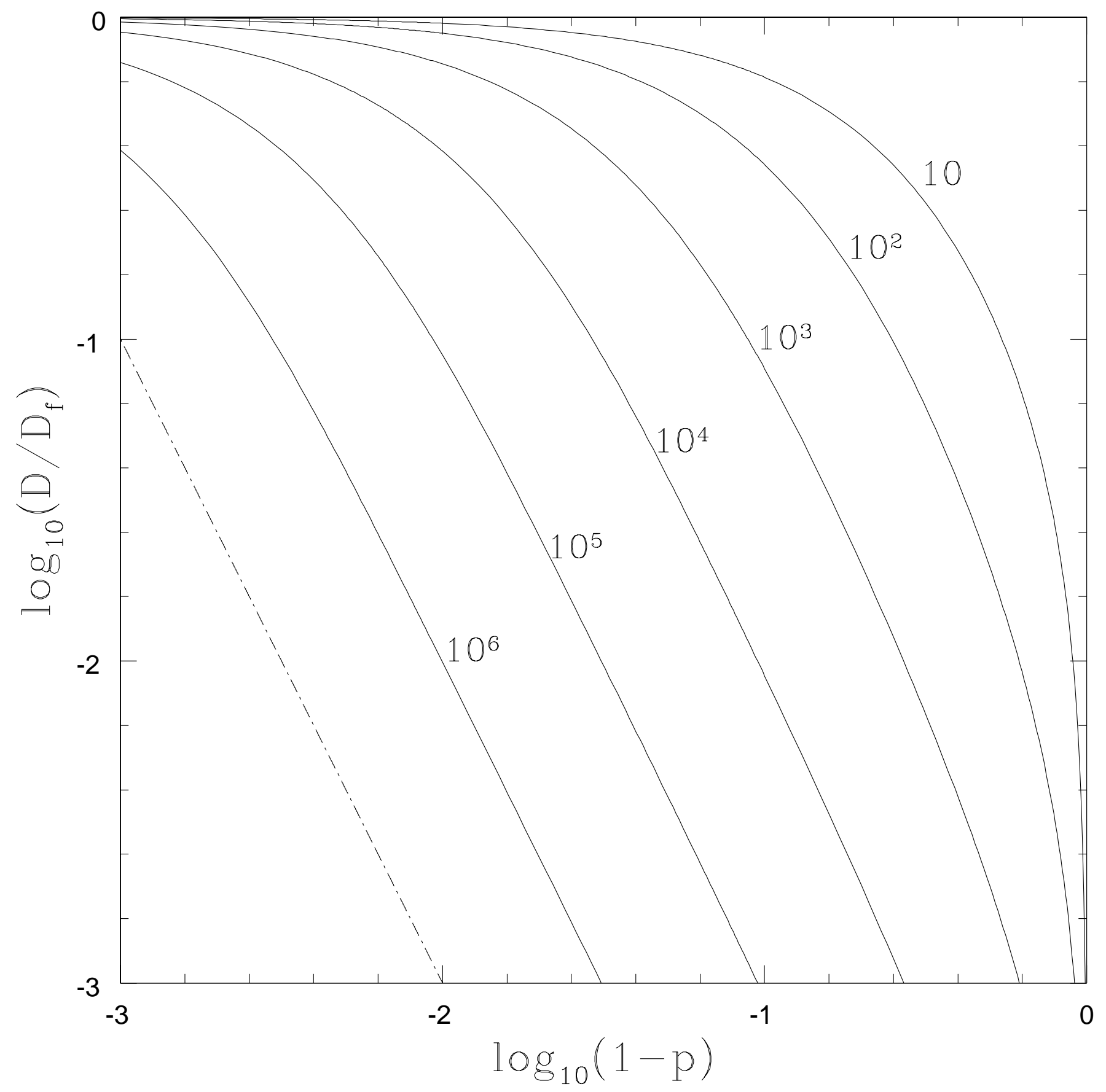

FIG. 1. The function $D / D_{f}$ is plotted versus $1-p$ at different values of $\tau_{s} / \tau_{f}$ in the double logarithmic scale. The function $(1-p)^{-2}$ is shown by dot-dashed staight line. 


\section{REFERENCES}

[1] D. Stauffer and A. Aharony, Introduction to Percolation Theory, Taylor and Frances, London, 1994.

[2] A. L. Efros, Phys. Rev. Lett. 682208 (1992.)

[3] A. L. Efros, D. Menashe, O. Biham, and B. D. Laikhtman, Phys. Stat.Sol. (b), 218,17 (2000).

[4] A. P. Chatterjee and R. F. Loring, Phys. Rev. E 50,2439 (1994)

[5] S. D. Druger, A. Nitzan, M. Ratner, J. Chem. Phys. 79, 3133 (1983).

[6] A. L. Efros, B. I. Shklovskii, Phys. Stat. Sol.,(b) 76,476 (1976).

[7] J. P. Straley, Phys. Rev B 15, 5733 (1977). 